\title{
Successful anesthesia in a patient with a history of cardiac arrest following rocuronium injection
}

\author{
Woosuk Chung, Dale Jang, Sang-Il Park, Won Hyung Lee, and Seok-Hwa Yoon \\ Department of Anesthesiology and Pain Medicine, Chungnam National University Hospital, Daejeon, Korea
}

There are now several reports of anaphylaxis after rocuronium injection in the Korean population, where only mild to moderate symptoms have been described. This is fortunate, since severe anaphylactic reactions have also been reported after rocuronium injection. Also, among the cases reported in Korea, only one case performed general anesthesia with a neuromuscular blocking agent later on [1]. Hence, we report a case of severe anaphylaxis after injection of rocuronium where the patient showed abrupt severe hypotension and cardiac arrest. After being transferred to the intensive care unit (ICU), ST segment depression was discovered in multiple electrocardiogram (ECG) leads and immediate coronary angiography revealed variant angina. After careful evaluation of the patient's severe anaphylactic reaction, the patient was successfully anesthetized four weeks later.

An 82-year-old male (height $169 \mathrm{~cm}$, weight $67 \mathrm{~kg}$ ) without any past medical history was scheduled for elective laparoscopyassisted distal gastrectomy. Although the preoperative ECG showed several premature ventricular contractions per minute, echocardiography results were normal. During induction, there was a sudden drop in blood pressure (BP) after rocuronium injection. Endotracheal intubation was immediately performed, and although there was no increase in airway resistance, BP continued to be severely hypotensive. The patient did not respond to intravenous ephedrine $(10 \mathrm{mg})$ or phenylephrine $(100 \mu \mathrm{g})$. Three minutes after the onset of hypotension, skin rash appeared over the entire body in addition to swelling of the eyes. As hemodynamics continued to deteriorate, epinephrine $(200 \mu \mathrm{g})$ was administered and cardiopulmonary resuscitation (CPR) was initiated. Rapid crystalloid fluid infusion was given after inserting a central venous catheter in the left subclavian vein. CPR was continued for 15 minutes and epinephrine (1 mg) was injected twice over a three-minute period. The patient was also defibrillated due to one episode of ventricular fibrillation during CPR. Hydrocortisone $(250 \mathrm{mg}$ ) and chlorpheniramine $(4 \mathrm{mg})$ were also injected, while epinephrine and dopamine were continuously infused. The patient was transferred to the ICU after full recovery of consciousness and muscle tone. Twenty-four hours after the event, cardiac enzymes had elevated (Troponin I 5.24 $\mathrm{ng} / \mathrm{ml}, \mathrm{CK}-\mathrm{MB} 23.5 \mathrm{ng} / \mathrm{ml}$ ) and ST depression could be seen in the I, aVL, and V2-6 leads of the ECG. Coronary angiography was immediately performed, and the patient was diagnosed with variant angina. Fortunately, the patient recovered without any complications and the operation was delayed by four weeks. Intradermal injection tests were performed twice: at three weeks and at four weeks after the event (Fig. 1). Due to the positive results with rocuronium and vecuronium, cisatracurium was used instead. Intravenous injection of chlorpheniramine $(4 \mathrm{mg})$ was added as premedication. There was no particular event during the second induction period, and the patient was discharged one week later.

Anaphylactic reaction can affect multiple organs, and although most patients recover spontaneously from mild reactions, respiratory and cardiovascular arrest can occur in a matter of minutes in severe cases. Reitter et al. [2] reported several factors associated with severe symptom: emergency operation, elderly and obese patients, history of hypertension, other cardiovascular disease and/or beta-blocker use. We believe that multiple factors may have caused cardiac arrest during induction

Corresponding author: Seok-Hwa Yoon, M.D., Ph.D., Department of Anesthesiology and Pain Medicine, Chungnam National University Hospital, 282, Munhwa-ro, Jung-gu, Daejeon 301-721, Korea. Tel: 82-42-280-7840, Fax: 82-42-280-7968, E-mail: seohwy@cnu.ac.kr (c) This is an open-access article distributed under the terms of the Creative Commons Attribution Non-Commercial License (http:// creativecommons.org/licenses/by-nc/3.0/), which permits unrestricted non-commercial use, distribution, and reproduction in any medium, provided the original work is properly cited. 
A

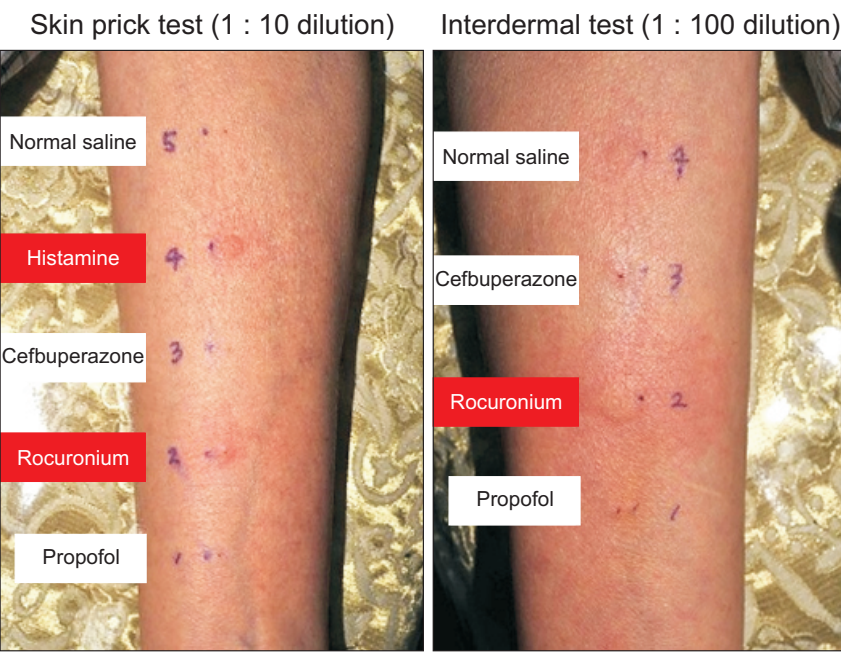

3 weeks after anaphylactic event
C

Skin prick test (1: 10 dilution)

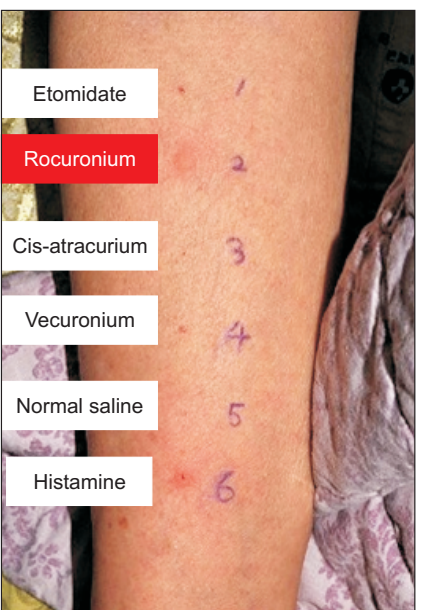

4 weeks after anaphylactic event
D

Interdermal test (1: 100 dilution)

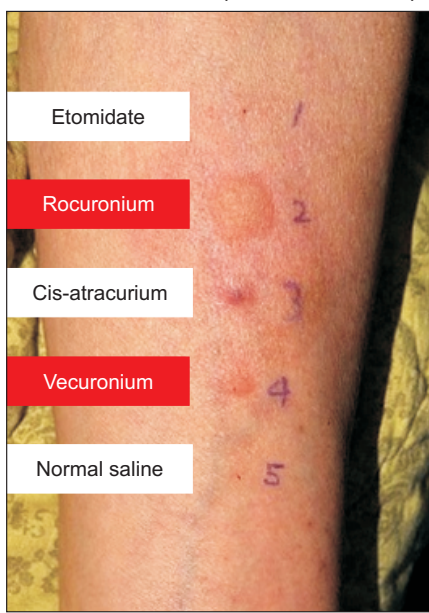

Fig. 1. Skin prick and intradermal test performed after three weeks and four weeks after the anaphylactic event. (A, B) Skin prick and intradermal test results at three weeks. Only rocuronium shows positive results. (C, D) Skin prick and intradermal test results at four weeks. Vecuronium also shows positive results in the intradermal test.

with rocuronium in the patient described here.

During evaluation after the anaphylactic event, variant angina was detected during cardio-angiography. Secondary acute coronary vasospasm after anaphylaxis was first reported in 1991 and has been termed the 'Kounis syndrome'. The possibility of Kounis syndrome after rocuronium injection was first reported by Fagley et al. [3]. In our present case, there was a significant increase in cardiac enzymes, ST depression was observed in multiple ECG leads and the patient was diagnosed with variant angina through coronary angiography. Although factors such as severe hypotension, ventricular fibrillation and CPR could have contributed to the development of variant angina, we cannot rule out the possibility of Kounis syndrome as the cause of coronary vasospasm. Even though we did not discover changes in the ST segment directly after rocuronium injection, this could have been due to limited ECG monitoring. Only the II lead was monitored during induction, while ST depression was found in the I, aVL and V2-6 leads in the ICU.

Other factors such as an unusually prolonged fasting period and a relatively high dose of propofol may also affect the clinical outcome of the patient. The patient had received upper gastrointestinal endoscopy and an enema the previous day. This led to a significantly prolonged fasting time since the patient had to continue fasting for the following operation. Indeed, extremely low central venous pressure $(0-2 \mathrm{mmHg}$ ) was observed during the event. Since anesthetics, especially propofol, affect the cardiovascular system more significantly in geriatric patients [4], the hypovolemic status combined with the relatively large dose of propofol may have contributed to the severity of the anaphylactic attack.

The decision was made to delay the operation by four weeks. Cisatracurium was selected as the neuromuscular blocking agent due to negative results during the skin prick test and intradermal test (Fig. 1). Although some recommend a period of six weeks after the anaphylactic event, tests were performed early due to the patients' strong desire for an early operation [5].

In conclusion, anesthesiologists must always pay special attention during the induction period due to the administration of various drugs over a relatively short period. Although anesthesiologists are skilled in immediate treatment during severe anaphylaxis, they must be aware of factors that could aggravate such physiological conditions. Also, even though extremely rare, anesthesiologists must also be aware of serious complications such as Kounis syndrome. 


\section{References}

1. Hwang SM, Suh JW, Lim SY. Anaphylaxis after thiopental and rocuronium induction: a case report. Korean J Anesthesiol 2005; 48: 95-8.

2. Reitter M, Petitpain N, Latarche C, Cottin J, Massy N, Demoly P, et al. Fatal anaphylaxis with neuromuscular blocking agents: a risk factor and management analysis. Allergy 2014; 69: 954-9.

3. Fagley RE, Woodbury A, Visuara A, Wall M. Rocuronium-induced coronary vasospasm--"Kounis syndrome". Int J Cardiol 2009; 137: e29-32.

4. Berthoud MC, McLaughlan GA, Broome IJ, Henderson PD, Peacock JE, Reilly CS. Comparison of infusion rates of three iv anaesthetic agents for induction in elderly patients. Br J Anaesth 1993; 70: 423-7.

5. Currie M, Kerridge RK, Bacon AK, Williamson JA. Crisis management during anaesthesia: anaphylaxis and allergy. Qual Saf Health Care 2005; 14: e19. 